\title{
The impairment of small nerve fibers in severe sepsis and septic shock
}

\author{
Hubertus Axer ${ }^{1 *}$ D, Alexander Grimm ${ }^{1,2}$, Christine Pausch ${ }^{3,4}$, Ulrike Teschner ${ }^{1}$, Jan Zinke ${ }^{1}$, Sven Eisenach', \\ Sindy Beck', Orlando Guntinas-Lichius ${ }^{5}$, Frank M. Brunkhorst ${ }^{6}$ and Otto W. Witte ${ }^{1}$
}

\begin{abstract}
Background: A decrease of small nerve fibers in skin biopsies during the course of critical illness has been demonstrated recently. However, the diagnostic use of skin biopsies in sepsis and its time course is not known.

Methods: Patients ( $n=32$ ) with severe sepsis or septic shock were examined using skin biopsies, neurological examination, nerve conduction studies, and sympathetic skin response in the first week after onset of sepsis, 2 weeks and 4 months later and compared to gender- and age-matched healthy controls.
\end{abstract}

Results: Skin biopsies at the ankle and thigh revealed a significant decrease of intraepidermal nerve fiber density (IENFD) during the first week of sepsis and 2 weeks later. All patients developed critical illness polyneuropathy (CIP) according to electrophysiological criteria and 11 showed IENFD values lower than the 0.05 quantile. Four patients were biopsied after 4 months and still showed decreased IENFD. Results of nerve conduction studies and IENFD did considerably change over time. No differences for survival time between patients with IEFND lower and larger than 3.5 fibers $/ \mathrm{mm}$ were found.

Conclusions: Skin biopsy is able to detect an impairment of small sensory nerve fibers early in the course of sepsis. However, it may not be suited as a prognostic parameter for survival.

Trial registration: German Clinical Trials Register, DRKS-ID: DRKS00000642, 12/17/2010

Keywords: Sepsis, Critical illness polyneuropathy, Skin biopsy, Small nerve fiber neuropathy

\section{Background}

Severe sepsis and septic shock represent the third leading cause of death in industrialized countries $[1,2]$ and are among the most challenging conditions in modern medicine. A particularly harmful complication of sepsis is ICU-acquired weakness (ICUAW) [3, 4]. ICUAW may be caused by muscle wasting [5], critical illness polyneuropathy (CIP), critical illness myopathy (CIM), or as in most cases, a combination of CIP and CIM [6]. About $70 \%$ of patients with sepsis [7], and up to $100 \%$ of patients with multi-organ failure develop CIP [8], but this may be an overestimation due to use of different diagnostic criteria between studies [9]. Development of CIP is associated with increased mortality, prolonged times on the respirator, and longer rehabilitation periods [10-12].

\footnotetext{
* Correspondence: hubertus.axer@med.uni-jena.de

${ }^{1}$ Hans Berger Department of Neurology, Jena University Hospital, Erlanger

Allee 101, D-07747 Jena, Germany

Full list of author information is available at the end of the article
}

CIP and CIM share the major clinical signs comprising symmetric and flaccid weakness of the muscles and the absence of deep tendon reflexes [13]. Patients with CIP also have distal loss of sensitivity to pain, temperature, and vibration. Nerve conduction studies detect CIP and CIM early during the first week of the disease $[14,15]$. Typical signs of CIP and CIM are reduction in the amplitude of compound muscle action potentials, whilst nerve conduction velocity is mainly preserved. In CIP, amplitudes of sensory nerve action potential (SNAP) may also be reduced or missing, indicating axonal damage to sensory nerves $[16,17]$.

In recent years skin biopsy has become a valuable tool for the evaluation of patients with small nerve fiber pathological change $[18,19]$, with reliable normative data [20]. Skin biopsy obtained using a disposable 3$\mathrm{mm}$-diameter punch is minimally invasive and generally safe [21]. Skin biopsy is typically taken at the ankle and/ or the thigh. The tissue is processed histologically with 
staining of intraepidermal nerve fibers. Nerve fiber density can be estimated by counting the nerve fibers crossing the basal lamina of the epidermis over the surface length of $50-\mu \mathrm{m}$-thick sections of the skin. Intraepidermal nerve fiber density (IENFD) closely correlates with clinical symptoms and is more sensitive than sensory nerve conduction studies and sural nerve biopsies for diagnosing small fiber neuropathy. Diagnostic efficiency and predictive values are very high using this technique $[18,22]$.

An impairment of small nerve fibers in sepsis may play a major pathophysiological role in neuropathic pain syndromes, which often become apparent after recovery from severe illness and after discharge from the ICU [23]. In addition, autonomic dysregulation plays a significant role in the acute phase of severe sepsis [24]. Lately, the occurrence of CIP has been related to decreased heart rate variability [25]. It is well-known that autonomic dysregulation is associated with increased long-term mortality rates $[26,27]$. In addition, there appears to be correlation between IENFD and abnormal autonomic function assessed by quantitative sudomotor axonal reflex test in patients with painful neuropathy [28]. Therefore, we hypothesized that impairment of small nerve fibers plays a role as a prognostic marker in sepsis.

Latronico et al. [29] performed skin biopsies at the ankle and thigh in 14 adult patients under neurocritical care, who had a prolonged ICU length of stay and artificial ventilation on median ICU-day 22. All patients had severe and non-length-dependent loss of intraepidermal nerve fibers. Of these patients 13 had infections, sepsis or multiple organ failure. In addition, sweat gland innervation was reduced in all except one patient.

In another study [30], IENFD of the distal leg was assessed on admission to the ICU and 10-14 days later. Of the 11 patients recruited, 9 (82\%) had sepsis or multiple organ failure. Median IENFD on admission decreased significantly. Abnormal IENFD was observed in 8 patients (72.7\%). Electrodiagnostic signs of large fiber neuropathy and/or myopathy were found in 6 patients (54.5\%), and autonomic dysfunction in 2 patients (18.2\%).

The aim of this study was to examine the decrease in intraepidermal nerve fibers in CIP in the early phase of sepsis, to describe the time course of intraepidermal nerve fiber density at the ankle and thigh during the course of sepsis, and to evaluate the use of IENFD as a prognostic marker in the acute phase of sepsis.

\section{Methods}

\section{Study design}

The study protocol for this single-center, non-randomized, controlled, observational study has been published earlier in detail [31]. The study has been registered with the German Clinical Trials Register (DRKS-ID: DRKS00000642).
Patients $\geq 18$ years of age with severe sepsis and/or septic shock according to published criteria [32] were screened for eligibility. Exclusion criteria were: history of neuromuscular disorders (such as polyneuropathy, myasthenia gravis, myopathy, and others), known alcohol abuse, high-dose steroid therapy before sepsis $(\geq 16 \mathrm{mg}$ prednisolone/kg body weight for 5 days or equivalent dose of other corticosteroids), ICU stay $\geq 8$ days, participation in another clinical study, platelet count $<40 \mathrm{Gpt} /$ $1\left(10^{9} / \mathrm{l}\right)$, partial thromboplastin time (PTT) $>60 \mathrm{~s}$, international normalized ratio (INR) $>1.7$, or if the patient was likely to die within less than 24 hours.

Patients admitted to the ICUs at Jena University Hospital between February 2011 and April 2015 were enrolled in the study. Written informed consent was obtained from all patients or their legal representatives and from all healthy controls. The study was approved by the Ethics Committee of the Friedrich Schiller University Jena (number 2771-02/10).

Baseline characteristics of the patients (e.g., underlying disease, age, gender, body mass index, ICU characteristics, i.e., duration of stay, length of mechanical ventilation, etc.) were obtained from the standardized prospective sepsis registry established at Jena University Hospital [33]. Study visits were performed between days 2 and 5 after onset of severe sepsis/septic shock, and at 2 weeks and 4 months, with skin biopsies performed at the thigh and ankle. Neurological examination (including the Medical Research Council (MRC) score for muscle strength), nerve conduction studies, and sympathetic skin response were undertaken at each visit. Figure 1 summarizes patient flow and study visits.

In addition, gender- and age-matched healthy controls with a normal neurological status were visited once and skin biopsies were taken from the right ankle and thigh. The age of the matched control was not permitted to differ by more than 2 years from the age of the consecutive patient. As the controls were required to be healthy (including the older individuals) exclusion criteria were defined as: diabetes mellitus, history of neuromuscular disorders (such as polyneuropathy, myasthenia gravis, myopathy, and others), alcohol abuse, infection, history of cancer, coagulopathy, or contraindication to local anesthetics. Healthy controls were recruited in the local sport clubs to specifically include healthy people of different ages.

\section{Nerve conduction studies}

Standardized nerve conduction studies were carried out at each visit using a portable electrophysiologic device (Synergy 15.0; Natus Europe GmbH, Planegg, Germany) with surface electrodes, according to standard procedures described in the literature [34]. The right median nerve, the right tibial nerve, the left peroneal nerve, and 


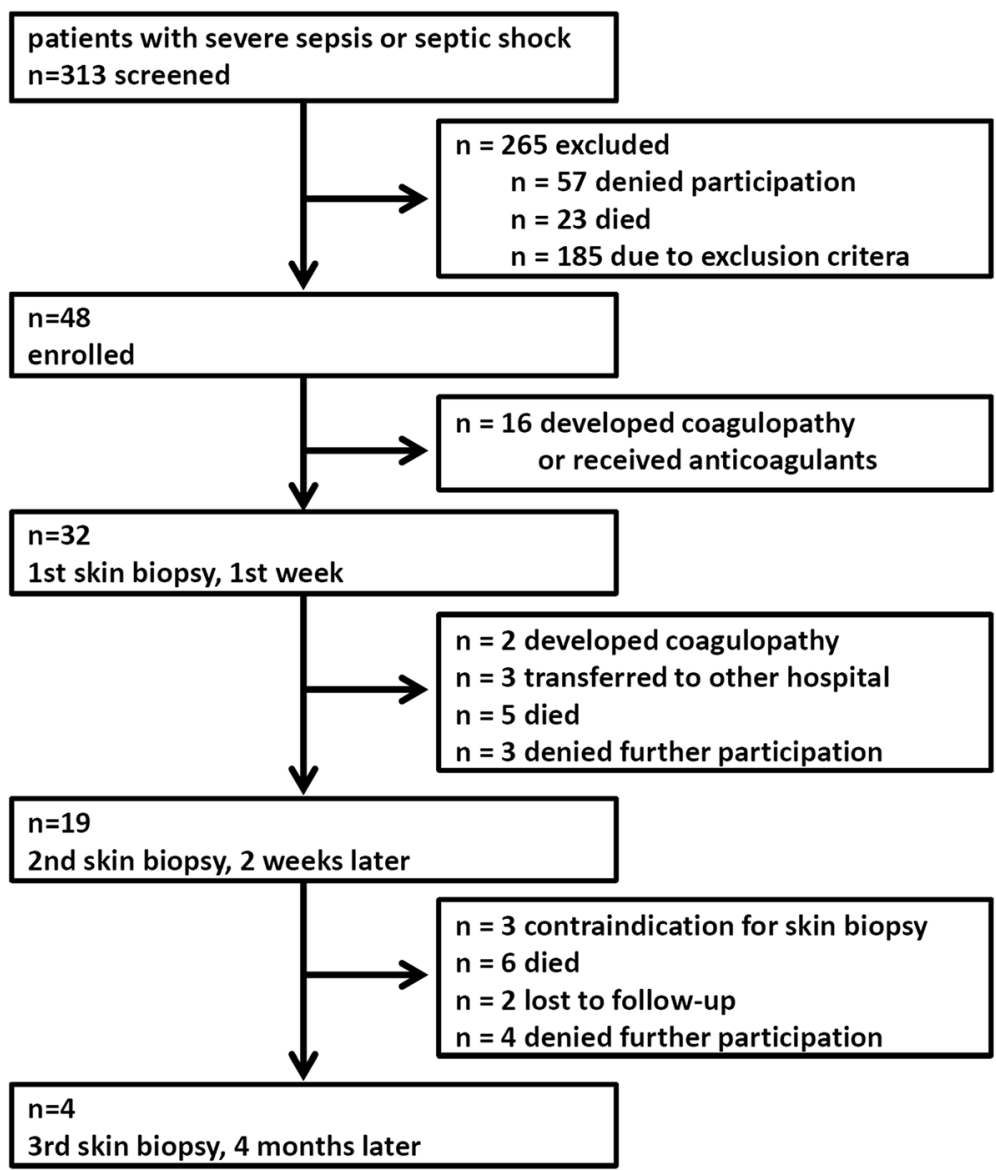

Fig. 1 Study flow

both sural nerves were measured. Motor and sensory nerve responses were assessed for the right median nerve, motor responses only for the tibial and fibular nerves, and sensory nerve responses for both sural nerves. This pattern was chosen for pragmatic reasons to guarantee a fast and easy electrophysiological checkup: three motor and three sensory measurements of the arm and both legs were obtained.

Sympathetic skin responses evoked by electrical stimulation of the median nerve were measured at the hands and feet as a neurophysiological equivalent of autonomic nerve function. Active surface electrodes were placed on the palmar side of the hands and the plantar side of the feet with reference electrodes on the dorsum of the hands and feet. An electrical stimulus $(20-30 \mathrm{~mA}, 0.2 \mathrm{~ms}$, with an irregular interval) was given to the right median nerve at the wrist. Skin potentials were recorded for a 10-s analysis period at both hands and feet. Sympathetic skin response was considered absent if there was no response after four stimuli.

Electrophysiological measurements were performed by experienced technical assistants from the neurophysiological department. Normative values for selected conduction study parameters derived from our own laboratory were used. Normative values were adjusted to age (e.g., $3.8 \mu \mathrm{V}$ for older age), as sural nerve SNAP is often much lower in older healthy individuals.

\section{Skin biopsies}

Skin biopsies were taken from the right ankle and thigh at each visit using a 3-mm biopsy punch and were immediately fixed in Zamboni's solution ( $2 \%$ paraformaldehyde and picric acid) for approximately $24 \mathrm{~h}$ at $4{ }^{\circ} \mathrm{C}$, then kept in a cryoprotective solution, and serially cut with a freezing microtome into $50-\mu \mathrm{m}$-thick sections. The sections were immunostained with anti-PGP 9.5 antibodies (anti protein gene product 9.5 antibody, ab72911, Abcam, Cambridge, UK) to label small nerve fibers in the skin and were counterstained with hematoxylin and eosin. Bright field microscopy for routine diagnostic purposes was used to count intraepidermal nerve fiber density (IENFD) defined by the number of stained nerve fibers crossing the basal lamina of the skin divided by the length of the epidermal surface. Image J $1.47 \mathrm{v}$ (National Institutes of Health, USA) was used for image processing [35]. 
Table 1 Demographics and baseline characteristics of patients $(n=32)$

\begin{tabular}{|c|c|}
\hline Parameter Statistic & \\
\hline \multicolumn{2}{|l|}{ Sociodemographic data } \\
\hline Age, years, median, mean (IQR) & $69,67.41(61.50-77.25)$ \\
\hline Male gender, number (\%) & $19(59.4)$ \\
\hline Body mass index, $\mathrm{kg} / \mathrm{m}^{2}$, mean $\pm \mathrm{SD}$ & $27.50 \pm 5.04$ \\
\hline \multicolumn{2}{|l|}{ Clinical data } \\
\hline $\mathrm{APACHE} \|$ score $^{\mathrm{a}_{r}}$ mean $\pm \mathrm{SD}$ & $23.38 \pm 5.73$ \\
\hline Septic shock, number (\%) & $25(78.1)$ \\
\hline History of diabetes, number (\%) & $13(40.6)$ \\
\hline Heart failure, number (\%) & $5(15.6)$ \\
\hline Cerebrovascular disease, number (\%) & $15(46.9)$ \\
\hline Renal dysfunction, number (\%) & $3(9.4)$ \\
\hline $\begin{array}{l}\text { Chronic obstructive pulmonary disease, } \\
\text { number (\%) }\end{array}$ & $4(12.5)$ \\
\hline Liver cirrhosis, number (\%) & $0(0.0)$ \\
\hline History of cancer ${ }^{\mathrm{b}}$, number (\%) & $2(6.3)$ \\
\hline Immunosuppressionc, number (\%) & $4(12.5)$ \\
\hline \multicolumn{2}{|l|}{ Recent surgical history } \\
\hline Elective surgery, number (\%) & $2(6.3)$ \\
\hline Emergency surgery, number (\%) & $8(25.0)$ \\
\hline No history of surgery, number (\%) & $22(68.8)$ \\
\hline \multicolumn{2}{|c|}{ Site of infection (multiple responses per patient possible) } \\
\hline Pneumonia, number (\%) & $20(62.5)$ \\
\hline Other respiratory tract, number (\%) & $3(9.4)$ \\
\hline Intraabdominal/gastrointestinal, number (\%) & $6(18.8)$ \\
\hline Bones/soft tissue, number (\%) & $0(0.0)$ \\
\hline Surgical wound infection, number (\%) & $0(0.0)$ \\
\hline Urogenital, number (\%) & $1(3.1)$ \\
\hline Primary bacteremia, number (\%) & $1(3.1)$ \\
\hline Cardiovascular, number (\%) & $1(3.1)$ \\
\hline $\begin{array}{l}\text { Others (central nervous system, thoracal, } \\
\text { catheter, etc.), number (\%) }\end{array}$ & $4(12.5)$ \\
\hline \multicolumn{2}{|l|}{ Source of infection } \\
\hline Community acquired, number (\%) & $9(28.1)$ \\
\hline Nosocomial, number (\%) & $23(71.9)$ \\
\hline \multicolumn{2}{|l|}{ Infection } \\
\hline Microbiologically confirmed, number (\%) & $27(84.4)$ \\
\hline Clinical evidence, number (\%) & $5(15.6)$ \\
\hline \multicolumn{2}{|c|}{ Mortality, length of stay, mechanical ventilation, renal replacement } \\
\hline ICU mortality, number (\%) & $2(6.3)$ \\
\hline Hospital mortality, number (\%) (95 \% Cl) & $5(15.6)(6.86 ; 31.75)$ \\
\hline $\begin{array}{l}\text { Length of stay on ICU, calendar days, } \\
\text { median (IQR) }\end{array}$ & $24.5(17-30.25)$ \\
\hline $\begin{array}{l}\text { Length of stay in hospital, calendar days, } \\
\text { median (IQR) }\end{array}$ & $29(24-48.5)$ \\
\hline Mechanical ventilation, number (\%) & $31(96.9)$ \\
\hline
\end{tabular}

Table 1 Demographics and baseline characteristics of patients $(n=32)$ (Continued)

\begin{tabular}{|c|c|}
\hline $\begin{array}{l}\text { Days with mechanical ventilation }{ }^{d} \text {, } \\
\text { median (IQR) }\end{array}$ & $20.5(14-29)$ \\
\hline Renal replacement therapy, number (\%) & $5(15.6)$ \\
\hline $\begin{array}{l}\text { Days with renal replacement therapy, } \\
\text { median (IQR) }\end{array}$ & $18(18-20)$ \\
\hline
\end{tabular}

Histological skin sections were independently analyzed by two trained raters (HA and SB) blinded for the assignment of the probe to either patient or control group. Intra-rater intraclass correlation coefficients (ICC) for estimation of IENFD were 0.977 at the ankle and 0.927 at the thigh. Inter-rater ICCs for estimation of IENFD at the ankle were 0.927 and 0.844 at the thigh. Data were reported from the first rater (HA). IEFND values were regarded as significantly reduced if they were lower than the 0.05 quantile per age span and gender according to a worldwide normative reference study [20].

\section{Statistics}

IBM SPSS Statistics Version 19 (SPSS Inc., Chicago, IL, USA) and R Version 3.1.0 [36] were used for statistical analysis. Descriptive statistics were used to characterize demographic data, baseline characteristics of the patients, and major parameters of nerve conduction studies such as amplitudes and conduction velocity. The unpaired $t$ test was used with a two-sided significance level of $5 \%$ to compare IENFD between groups. Kaplan-Meier curves and the log-rank test were used to analyze the use of IENFD at the ankle in the first week of sepsis as a possible prognostic factor for survival.

\section{Results}

The demographic data of our study population are presented in Table 1. 313 patients were screened, but in many cases $(18 \%)$ the patients' legal representatives did not approve participation in the study, mainly due to the invasive character of skin biopsy. Exclusion criteria were met in 185 patients (59\%), essentially the existence of polyneuropathy, alcohol abuse, or coagulopathy. A total of 32 patients, who fulfilled the criteria for severe sepsis or septic shock, were biopsied in the first week after onset of sepsis. Healthy controls $(n=32)$ with normal neurological status were matched to the sepsis patients according to age and gender (19 male and 13 female individuals in both groups, mean age 66.75 years, median age 71.5 years, IQR $60.5-75.0$ in the control group). Healthy controls did not have any metabolic disease (such as diabetes mellitus), neuromuscular disease, or alcohol abuse. They only had comorbidities that do not 
influence neuropathy (high blood pressure $(\mathrm{n}=4)$, coronary heart disease $(n=2)$, lower back pain $(n=2)$, depression $(\mathrm{n}=1)$, and history of basal cell carcinoma that had been completely excised and cured $(n=1)$ ).

All sepsis patients had CIP according to electrophysiological criteria. CIP was diagnosed, when amplitudes of compound muscle action potential and sensory nerve action potential $[17,37]$ were reduced in at least three nerves. Electrophysiological diagnosis of CIP was not based on pathological sural nerve potential alone as these may be notably susceptible to edema. Clinically, all patients suffered from muscle weakness (see Additional file 1). However, the majority of patients received analgo-sedation and mechanical ventilation (25 patients $(78.1 \%)$ with mechanical ventilation at first visit). None of the patients were exposed to high-dose steroids (exclusion criteria of the study) or neuromuscular blocking agents. No patient suffered from HIV. All patients received passive or active physiotherapy.

Nerve conduction studies displayed changes typical of axonal damage during the first week after onset of sepsis. Reductions in amplitudes of motor and sensory action potential were more pronounced than reductions in conduction velocity. Electrophysiological parameters did not change significantly over the time course of sepsis (see box plots of amplitudes of action potential and conduction velocity in Additional file 2). The sympathetic skin response at the hands and feet was lost in all patients.
Skin biopsies (Fig. 2) revealed a considerable decrease in small nerve fibers in the septic patients early in the course of disease. Eleven of the patients (34\%) showed IEFND values lower than the 0.05 quantile per age span and gender according to a worldwide normative reference study [20] during the first week of severe sepsis or septic shock. Figure 3 shows the boxplots for intraepidermal nerve fiber density at the ankle and thigh of the patients in comparison to the controls. IENFD at the ankle and thigh were significantly decreased $(p<0.0001)$ in patients compared to age- and gender-matched controls during the first week of sepsis $(\mathrm{n}=32)$ and 2 weeks later $(\mathrm{n}=19)$. After 4 months, four patients were subjected to a third skin biopsy. In these patients IEFND was still reduced. The results were not statistically significant ( $p=0.452$ at the ankle, $p=0.076$ at the thigh) due to the small sample size. The main reasons (Fig. 1) for not obtaining follow-up skin biopsies were death and the development of contraindications to skin biopsy, such as anticoagulation or coagulopathy.

The ratio between IENFD at the ankle and thigh did not differ between patients and controls $(p=0.103$ at visit $1, p=0.113$ at visit 2 , and $p=0.8$ at visit 3 ). This ratio is an indicator of length-dependent nerve damage; it is considerably increased if distal nerve fibers (at the ankle) are impaired to a much greater extent than proximal nerve fibers (at the thigh).

At the first visit only 7 of the 32 patients were evaluated for sensory symptoms such as sensory loss or dysesthesia. Mean pallesthesia was 3.7 (on a scale

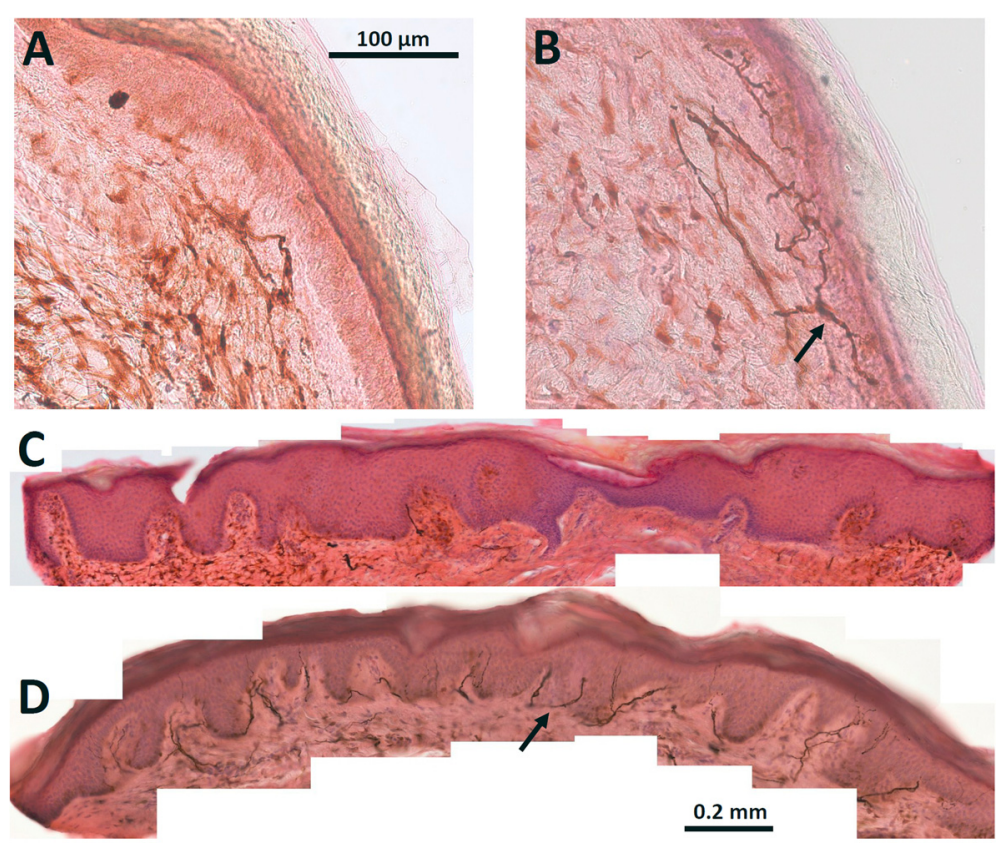

Fig. 2 Example of histology of skin biopsies in septic patients ( $\mathbf{a}$ and $\mathbf{c}$ ) and normal controls (b and $\mathbf{d}$ ). Intraepidermal nerve fiber density is considerably decreased in septic patients. Arrows show examples of nerve fibers 


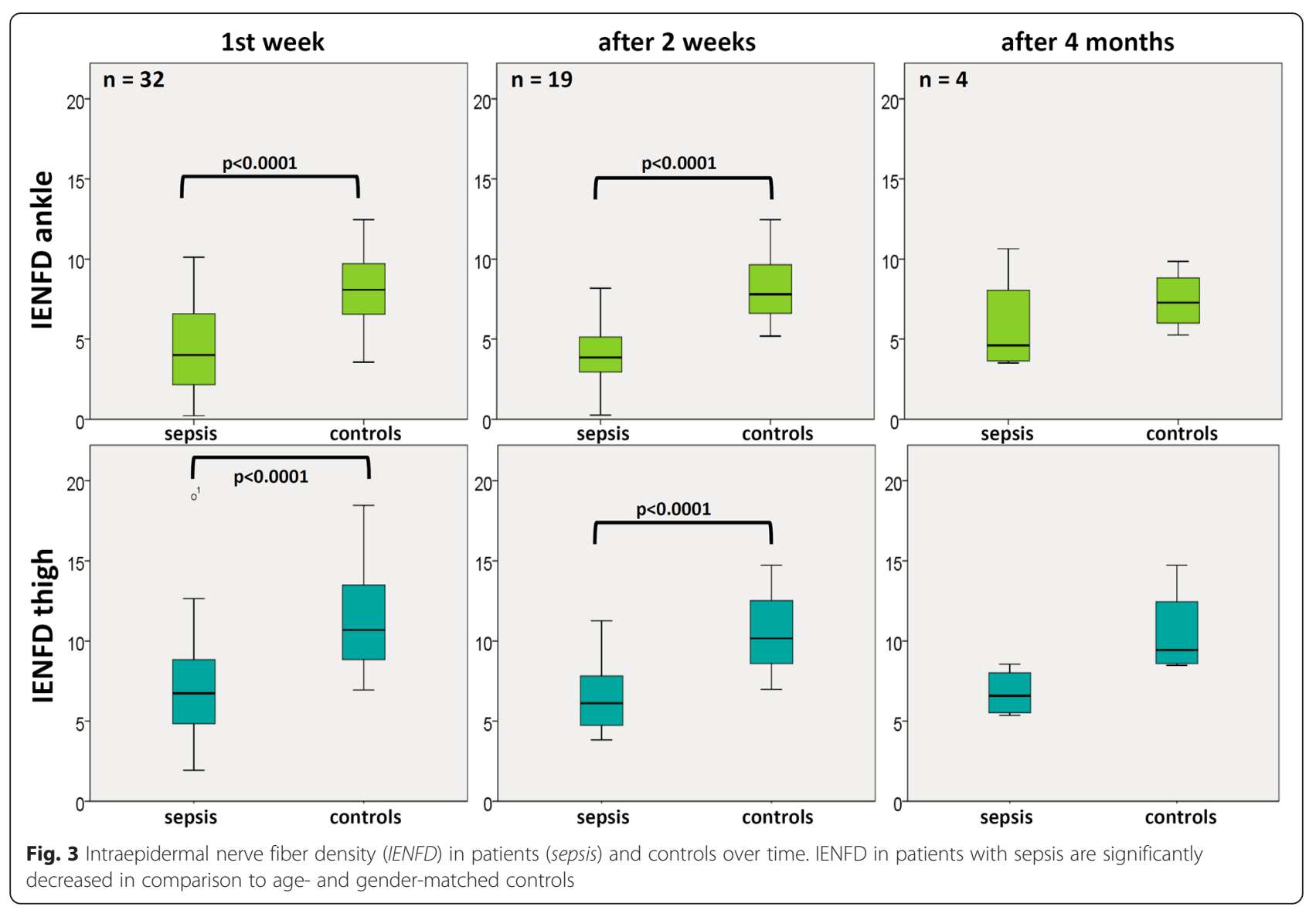

between 0 (pallanaesthesia) and 8 (normal pallaesthesia)) with standard deviation of 3.3 measured by the use of a tuning fork placed over the lateral malleolus. At visit 2 , there were 10 out of 19 patients evaluated: only 1 of the 10 patients complained of sensory symptoms. The four patients examined after 4 months had mean pallesthesia of 5.6 with standard deviation of 1.5. Three of these patients complained of sensory symptoms.

In addition, patients with sepsis were grouped according to the number of confounding co-morbidities for neuropathy (especially diabetes, history of cancer and chronic kidney disease, Table 1). Alcohol abuse was an exclusion criterion. IENFD at the ankle and thigh was significantly reduced in the 17 patients without confounding comorbidities in comparison to the healthy controls (Fig. 4).

Patients were divided into two groups comprising those with IEFND below, and those with IEFND >3.5 fibers $/ \mathrm{mm}$ of skin, to test whether in the acute phase of sepsis IENFD at the ankle is a prognostic marker for survival. On Kaplan-Meier curves there were no significant differences in survival time in the two groups ( $p=0.927$, Fig. 5). Median survival time was 444 days for patients with an IENFD $<3.5$ fibers $/ \mathrm{mm}$ and 303 days for patients with an IENFD $>3.5$ fibers $/ \mathrm{mm}$.

\section{Discussion}

Skin biopsy has become a valuable tool in the evaluation of patients with small nerve fiber pathological changes [21]. Skin biopsy represents a well-established, safe, easy, and inexpensive method to quantify small sensory nerve fibers and autonomic nerve fibers in the skin [18]. In contrast, nerve conduction studies are not able to evaluate the function of small nerve fibers. Damage to small nerve fibers has recently been demonstrated in small samples of critically ill patients [29, 30]. Small fiber pathological change may play a role in the pathophysiology of sepsis, e.g., in neuropathic pain syndromes, which often become apparent after recovery from severe illness and after discharge from the ICU [23]. Although sensory problems like neuropathic pain, stocking/glove sensory loss, numbness and pallhypesthesia were evaluated in this study, the majority of patients were not able to report these reliably in the acute phase of sepsis. As neuropathic pain is a problem in the long-term follow up of patients with sepsis, a study addressing this specific issue has to be planned using longer periods of follow up.

In addition, autonomic dysregulation plays a significant role in the acute phase of severe sepsis [24]. Lately, the occurrence of CIP has been related to decreased 

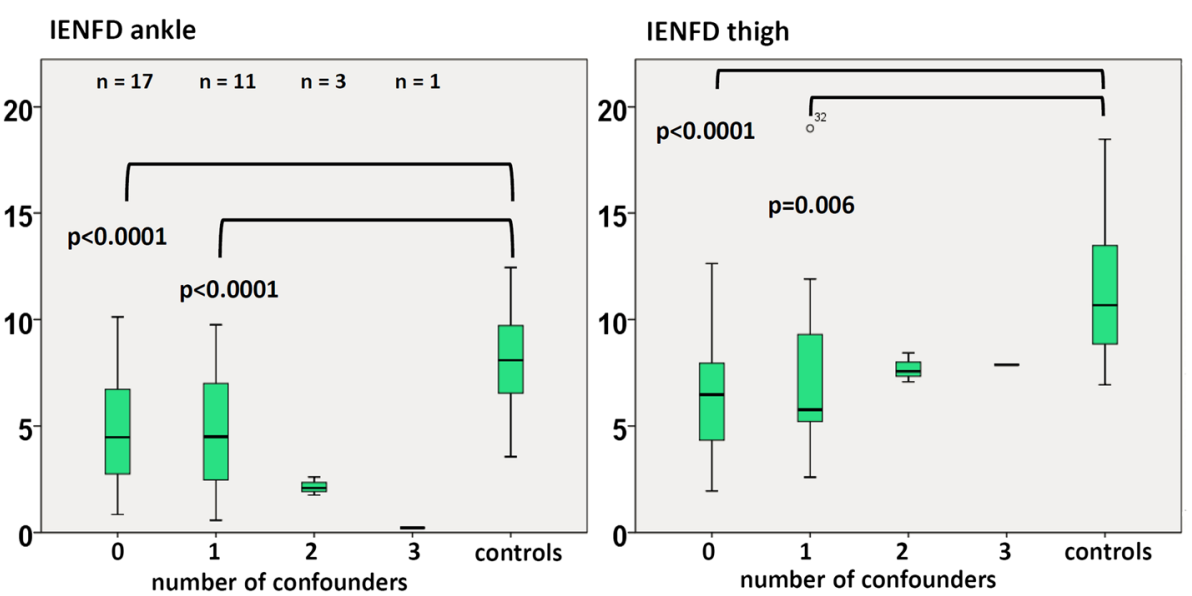

Fig. 4 Intraepidermal nerve fiber density (IENFD) over time in patients grouped according to number of confounding comorbidities for neuropathy. In patients without any confounding comorbidities $(n=17)$ there was significant loss of IENFD at ankle and thigh in comparison to the healthy controls

heart rate variability [25]. As autonomic dysregulation is associated with increased long-term mortality [26, 27], impairment of small nerve fibers may have an impact on the prognosis of patients with sepsis.

In our study all patients suffered from CIP according to electrophysiological criteria. All patients had loss of sympathetic skin response in the hands and feet. The latter observation may also be influenced by catecholamine therapy [38] and can therefore not be regarded as a secure electrophysiological sign of autonomic neuropathy.

A major finding of this study is the early decrease in IEFND during the first week of severe sepsis and septic shock. In the present study, the ratio of IENFD at the ankle (distal) and thigh (proximal) of patients and controls were not length-dependent. Length-dependent axonal

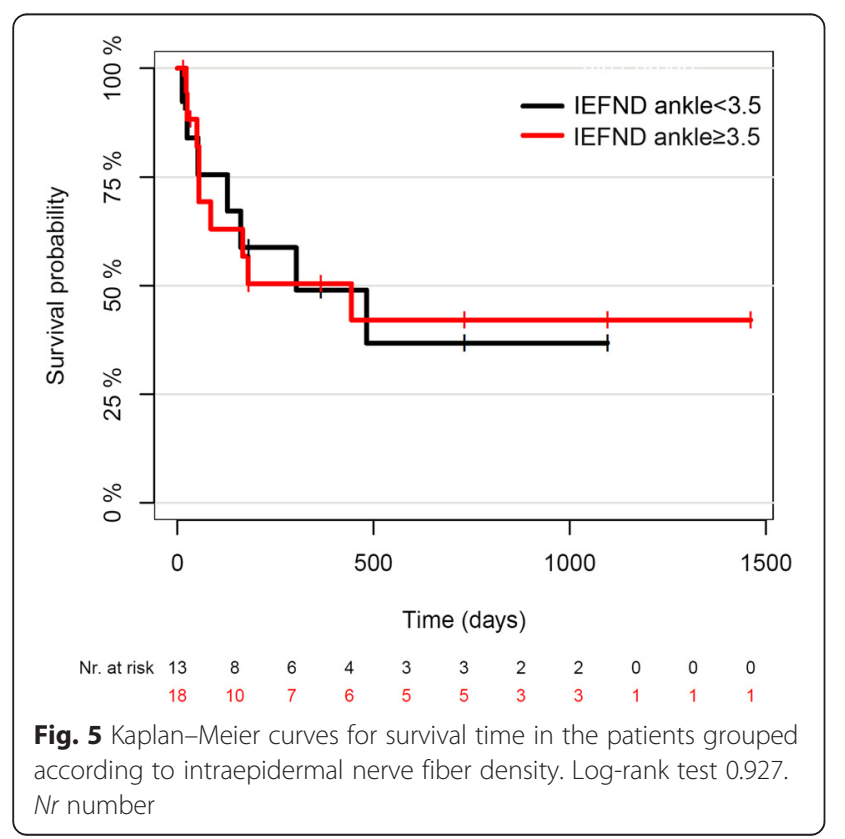

degeneration (the so-called dying-back phenomenon) results in a typically distal-to-proximal gradient of axonal degeneration with symmetrical and distally pronounced neuropathic symptoms.

The reduction in intraepidermal nerve fibers in our patients, who received the first biopsies on median day 4 after onset of sepsis, was similar to that observed by Latronico et al. [29], who performed skin biopsies on median ICU day 22. Skorna et al. [30] performed skin biopsies on the admission to the ICU and 10-14 days later. They demonstrated that IENFD decreased in 8 of 13 patients $(72.7 \%)$ after the admission to the ICU.

Although we excluded patients with known neuromuscular disorders such as polyneuropathy, one has to take into consideration that $40.6 \%$ of our patients suffered from diabetes, which is a risk factor for peripheral nerve damage. However, subgroup analysis showed that the 17 patients with sepsis who did not have confounding comorbidities for neuropathy also had decreased IENFD similar to those patients with one confounder for neuropathy, such as diabetes, chronic kidney disease, or history of cancer. To overcome this potential source of error, future studies should be based on patients admitted to the ICU with and without sepsis to study sepsis-specific neuropathy. All examinations of neuromuscular disturbance in sepsis have their own limitations. A reliable estimation of muscle strength in non-alert and non-awake patients is generally not possible. In many cases temporal interruption of sedative drugs is not possible in hemodynamically instable patients. Therefore, the MRC scores often cannot be reliably measured in the acute phase of sepsis.

Amplitudes of compound motor action potential and sensory nerve action potential in nerve conduction studies (NCS) may also be reduced due to edema, which is often a problem in sepsis. Moreover, NCS findings do not exclude myopathy. As CIP and CIM often occur together 
$[39,40]$, and muscle biopsies or direct muscle stimulation were not performed to distinguish CIP from CIM in this study, patients may also suffer from CIM. The difference is also important with respect to the prognosis, because the consequences of CIP last much longer than those of CIM [41], in terms of persistent muscle weakness and the development of chronic pain syndromes [29, 42]. In addition, repetitive nerve stimulation was not performed to rule out myasthenia-type abnormalities. Skin biopsy allows direct allocation of the damage to the peripheral nerves. It, therefore, may facilitate the differentiation between CIP and CIM. However, the study was not designed to address this research question. Although skin biopsy is an invasive method it causes only minimal pain using local anesthesia. The histological work-up and analysis of the skin biopsy takes some days, so that the result of the skin biopsy is not directly available at the bedside, which is a pragmatic restriction of the method.

Many of the legal representatives of the patients rejected the participation of the patient in the study because they feared the invasive character of skin biopsy. In addition, the study had a high drop-out rate of patients over time due to high mortality rates and contraindications against skin biopsy (such as coagulopathy or anticoagulation). Therefore, the initial target number of patients $(n=200)$ as designated in the study protocol [31] was not reached although over 300 patients were screened. However, the extent the decrease in IENFD was much larger than expected and the results were statistically significant based on considerably fewer patients than initially scheduled.

IEFND values did not change considerably over a longer time course. In addition, the time course of decrease of IEFND was similar to the time course of nerve conduction study abnormalities. This is consistent with a predominant axonal type of neuropathy with slow regeneration of the nerves (about $1 \mathrm{~mm}$ per day) which requires long-lasting rehabilitation. However, there are extreme biases related to the four patients who underwent repeat biopsies at 4 months. These biases unfortunately question the value of these results. Therefore, we cannot draw reliable conclusions from these results.

We also tested for IENFD in the first week of sepsis as a prognostic biomarker for survival, because autonomic neuropathy may cause autonomic dysregulation and leads to increased mortality rates [25, 27]. In a follow-up analysis of survival among our patients using the prospective sepsis registry, a clear prognostic value of IENFD at the ankle in the first week of sepsis was not be observed. The cutoff value of 3.5 fibers $/ \mathrm{mm}$ was arbitrarily chosen for practical reasons although IENFD normative values are age- and sex-dependent [20]. All 11 patients with an IEFND $<0.05$ quantiles per age span and gender were included in the group with an IEFND $<3.5$ fibers $/ \mathrm{mm}$ and their survival rate did not differ from that in the other groups. However, the sample size may have been too small to differentiate survivors from non-survivors. A larger study would be needed to definitely conclude that IEFND may not be suitable as a prognostic parameter for survival.

\section{Conclusions}

The density of small sensory nerve fibers in the skin is reduced early in the course of sepsis. This reduction does not depend on the length of the axons. The decrease in intraepidermal nerve fibers does not considerably change over time in the acute phase of sepsis; it is not a good predictor for survival.

\section{Key messages}

- Skin biopsy detects impairment of small sensory nerve fibers early in the course of sepsis

- It is not suitable as a prognostic marker for survival

- Intraepidermal nerve fiber density does not change considerably over the first weeks of sepsis

\section{Additional files}

Additional file 1: Medical Research Council (MRC) scores of muscle weakness over time. MRC scores of muscle strength were quantified bilaterally in the arms and legs. Figure shows examples of different proximal and distal movements of the limbs and MRC sum score. Note that the majority of patients could not reliably be evaluated due to analgo-sedation (2 patients with Richmond Agitation Sedation Scale (RASS) $<-1$ in the second week and mechanical ventilation (78\% of patients $(n=25)$ in the first week and $50 \%(n=9)$ in the second week), so that muscle strength could only be evaluated in 7 patients in the first week and 10 patients in the second week. In the patients examined after 4 months there was a considerable improvement in muscle strength, but muscle weakness was still detectable. Muscle weakness was always symmetrical and affected both proximal and distal muscle groups. (PDF $533 \mathrm{~kb}$ )

Additional file 2: Nerve conduction studies. Amplitudes of compound potentials in motor and sensory nerves are considerably decreased, and conduction velocity is marginally impaired. Note that nerve conduction studies detect critical illness polyneuropathy early in the course of sepsis, but amplitudes do not change considerably over the course of the disease. Horizontal lines show the normative values of the measurements. (PDF $340 \mathrm{~kb}$ )

\section{Abbreviations}

CIM: critical illness myopathy; CIP: critical illness polyneuropathy; ICU: intensive care unit; ICUAW: ICU-acquired weakness; IENFD: intraepidermal nerve fiber density; MRC: Medical Research Council; RASS: Richmond Agitation Sedation Scale; SNAP: sensory nerve action potential.

\section{Competing interests}

The authors declare that they have no competing interests.

\section{Authors' contributions}

AG participated in study design, acquisition of data and interpretation of data, and helped to draft the manuscript. UT and SB participated in the acquisition of data, were involved in the analysis of data, and revised the manuscript. CP performed the statistical analysis, was involved in the analysis and interpretation of data, and helped to draft the manuscript. JZ and SE participated in the acquisition of data, were involved in interpretation of data, and helped to revise the manuscript. FMB and OGL participated in the 
design of the study, were involved in the interpretation of data, and helped to revise the manuscript. OWW participated in the design of the study, was involved in the analysis and interpretation of data, and helped to draft the manuscript. HA conceived of the study, and participated in its design and coordination, was involved in the analysis and interpretation of data, and drafted the manuscript. All authors read and approved the final manuscript.

\section{Acknowledgements}

The study was supported by the Ministry of Education and Research (BMBF), grant number 01 E0 1002. We thank Nasim Krögel for help with language editing.

\section{Author details}

${ }^{1}$ Hans Berger Department of Neurology, Jena University Hospital, Erlanger Allee 101, D-07747 Jena, Germany. ${ }^{2}$ Department of Neurology and Epileptology, University of Tuebingen, Tuebingen, Germany. ${ }^{3}$ Center for Sepsis Control and Care (CSCC), Jena University Hospital, Jena, Germany. ${ }^{4}$ Institute for Medical Informatics, Statistics and Epidemiology (IMISE), University Leipzig, Leipzig, Germany. ${ }^{5}$ Department of Otorhinolaryngology, Jena University Hospital, Jena, Germany. ${ }^{6}$ Center for Clinical Studies, Jena University Hospital, Jena, Germany.

Received: 5 November 2015 Accepted: 13 February 2016 Published online: 15 March 2016

\section{References}

1. Engel C, Brunkhorst FM, Bone H-G, Brunkhorst R, Gerlach H, Grond S, et al. Epidemiology of sepsis in Germany: results from a national prospective multicenter study. Intensive Care Med. 2007;33:606-18.

2. Cawcutt KA, Peters SG. Severe sepsis and septic shock: clinical overview and update on management. Mayo Clin Proc. 2014;89:1572-8.

3. Kress JP, Hall JB. ICU-Acquired Weakness and Recovery from Critical IIIness. N Engl J Med. 2014;370:1626-35.

4. Fan E, Cheek F, Chlan L, Gosselink R, Hart N, Herridge MS, et al. An official American Thoracic Society Clinical Practice guideline: the diagnosis of intensive care unit-acquired weakness in adults. Am J Respir Crit Care Med. 2014;190:1437-46.

5. Puthucheary ZA, Rawal J, McPhail M, Connolly B, Ratnayake G, Chan P, et al. Acute skeletal muscle wasting in critical illness. JAMA. 2013;310:1591-600.

6. Zink W, Kollmar R, Schwab S. Critical illness polyneuropathy and myopathy in the intensive care unit. Nat Rev Neurol. 2009;5:372-9.

7. Visser LH. Critical illness polyneuropathy and myopathy: clinical features, risk factors and prognosis. Eur J Neurol. 2006;13:1203-12.

8. Hermans G, De Jonghe B, Bruyninckx F, Van den Berghe G. Clinical review: Critical illness polyneuropathy and myopathy. Crit Care. 2008;12:238.

9. Stevens RD, Dowdy DW, Michaels RK, Mendez-Tellez PA, Pronovost PJ, Needham DM. Neuromuscular dysfunction acquired in critical illness: a systematic review. Intensive Care Med. 2007;33:1876-91.

10. Garnacho-Montero J, Madrazo-Osuna J, García-Garmendia JL, Ortiz-Leyba C, Jiménez-Jiménez FJ, Barrero-Almodóvar A, et al. Critical illness polyneuropathy: risk factors and clinical consequences. A cohort study in septic patients. Intensive Care Med. 2001;27:1288-96.

11. Garnacho-Montero J, Amaya-Villar R, García-Garmendía JL, Madrazo-Osuna J, Ortiz-Leyba C. Effect of critical illness polyneuropathy on the withdrawal from mechanical ventilation and the length of stay in septic patients. Crit Care Med. 2005;33:349-54

12. Griffiths RD, Hall JB. Intensive care unit-acquired weakness. Crit Care Med. 2010;38:779-87.

13. Latronico N, Bolton CF. Critical illness polyneuropathy and myopathy: a major cause of muscle weakness and paralysis. Lancet Neurol. 2011;10:931-41.

14. Khan J, Harrison TB, Rich MM, Moss M. Early development of critical illness myopathy and neuropathy in patients with severe sepsis. Neurology. 2006;67:1421-5.

15. Latronico N, Bertolini G, Guarneri B, Botteri M, Peli E, Andreoletti S, et al. Simplified electrophysiological evaluation of peripheral nerves in critically ill patients: the Italian multi-centre CRIMYNE study. Crit Care. 2007;11:R11.

16. Axer H, Zinke J, Axer M, Grimm A. Electrophysiological assessment of critical illness polyneuropathy and critical illness myopathy. Neurophysiol Lab. 2013;35:18-33.

17. Bednarik J, Lukas Z, Vondracek P. Critical illness polyneuromyopathy: the electrophysiological components of a complex entity. Intensive Care Med. 2003;29:1505-14.
18. Joint Task Force of the EFNS and the PNS. European Federation of Neurological Societies/Peripheral Nerve Society Guideline on the use of skin biopsy in the diagnosis of small fiber neuropathy. Report of a joint task force of the European Federation of Neurological Societies and the Peripheral Nerve Society. J Peripher Nerv Syst. 2010;15:79-92.

19. Devigili G, Tugnoli V, Penza P, Camozzi F, Lombardi R, Melli G, et al. The diagnostic criteria for small fibre neuropathy: from symptoms to neuropathology. Brain. 2008;131:1912-25.

20. Lauria G, Bakkers M, Schmitz C, Lombardi R, Penza P, Devigili G, et al. Intraepidermal nerve fiber density at the distal leg: a worldwide normative reference study. J Peripher Nerv Syst. 2010;15:202-7.

21. Lauria G, Devigili G. Skin biopsy as a diagnostic tool in peripheral neuropathy. Nat Clin Pract Neurol. 2007;3:546-57.

22. Lauria G, Cornblath DR, Johansson O, McArthur JC, Mellgren SI, Nolano M, et al. EFNS guidelines on the use of skin biopsy in the diagnosis of peripheral neuropathy. Eur J Neurol. 2005;12:747-58.

23. Latronico N, Shehu I, Seghelini E. Neuromuscular sequelae of critical illness. Curr Opin Crit Care. 2005;11:381-90.

24. Annane D, Trabold F, Sharshar T, Jarrin I, Blanc AS, Raphael JC, et al. Inappropriate sympathetic activation at onset of septic shock: a spectral analysis approach. Am J Respir Crit Care Med. 1999;160:458-65.

25. Sagui E, Cordier PY, Nau A, Rogier C, Bregigeon M, Brosset C. Is critical illness polyneuropathy associated with decreased heart rate variability? Rev Neurol (Paris). 2014;170:32-6.

26. Schmidt H, Müller-Werdan U, Hoffmann T, Francis DP, Piepoli MF, Rauchhaus $\mathrm{M}$, et al. Autonomic dysfunction predicts mortality in patients with multiple organ dysfunction syndrome of different age groups. Crit Care Med. 2005;33:1994-2002.

27. Schmidt H, Hoyer D, Hennen R, Heinroth K, Rauchhaus M, Prondzinsky R, et al. Autonomic dysfunction predicts both 1- and 2-month mortality in middle-aged patients with multiple organ dysfunction syndrome. Crit Care Med. 2008:36:967-70.

28. Novak V, Freimer ML, Kissel JT, Sahenk Z, Periquet IM, Nash SM, et al. Autonomic impairment in painful neuropathy. Neurology. 2001;56:861-8.

29. Latronico N, Filosto M, Fagoni N, Gheza L, Guarneri B, Todeschini A, et al. Small nerve fiber pathology in critical illness. PLoS One. 2013;8:e75696.

30. Skorna M, Kopacik R, Vlckova E, Adamova B, Kostalova M, Bednarik J. Small nerve fiber pathology in critical illness documented by serial skin biopsies. Muscle Nerve. 2015;52:28-33.

31. Axer H, Grimm A, Porzelius C, Teschner U, Schumacher U, Witte OW, et al. Impairment of small somatic and autonomic nerve fibres in intensive care unit patients with severe sepsis and critical illness polyneuropathy - a single center controlled observational study. BMC Neurol. 2013;13:159.

32. Brunkhorst FM, Engel C, Bloos F, Meier-Hellmann A, Ragaller M, Weiler N, et al. Intensive insulin therapy and pentastarch resuscitation in severe sepsis. N Engl J Med. 2008;358:125-39.

33. Schmidt K, Gensichen J, Thiel P, Ludewig K, Bahr V, Rissner F, et al. A prospective observational web-based registry for assessment of long-term outcome in patients with severe sepsis. Infection. 2013;41:S84-5.

34. Preston D, Shapiro B. Electromyography and Neuromuscular Disorders, Clinical-Electrophysiological Correlations. New York: Elsevier Saunders; 2013.

35. Schneider CA, Rasband WS, Eliceiri KW. NIH Image to ImageJ: 25 years of image analysis. Nat Methods. 2012;9:671-5.

36. R Core Team: R: A language and environment for statistical computing. http://www.R-project.org. Accessed December 2014.

37. Bolton CF. Neuromuscular manifestations of critical illness. Muscle Nerve. 2005;32:140-63.

38. Storm H, Myre K, Rostrup M, Stokland O, Lien MD, Raeder JC. Skin conductance correlates with perioperative stress. Acta Anaesthesiol Scand. 2002;46:887-95.

39. Kerbaul F, Brousse M, Collart F, Pellissier J-F, Planche D, Fernandez C, et al. Combination of histopathological and electromyographic patterns can help to evaluate functional outcome of critical ill patients with neuromuscular weakness syndromes. Crit Care. 2004;8:R358-366.

40. Grimm A, Teschner U, Porzelius C, Ludewig K, Zielske J, Witte OW, et al. Muscle ultrasound for early assessment of critical illness neuromyopathy in severe sepsis. Crit Care. 2013;17:R227.

41. Koch S, Wollersheim T, Bierbrauer J, Haas K, Mörgeli R, Deja M, et al. Longterm recovery In critical illness myopathy is complete, contrary to polyneuropathy. Muscle Nerve. 2014;50:431-6.

42. Battle CE, Lovett $\mathrm{S}$, Hutchings $\mathrm{H}$. Chronic pain in survivors of critical illness: a retrospective analysis of incidence and risk factors. Crit Care. 2013;17:R101. 